\title{
The Importance of Maternal Thyroid for the Placental Function
}

Mirjana Barjaktarovic ${ }^{1,2,3}$, Tim I M Korevaar ${ }^{1,2,3}$, Vincent W V Jaddoe ${ }^{1,6,7}$, Yolanda B de Rijke ${ }^{2,5}$

Theo J Visser ${ }^{2,3}$, Eric A P Steegers ${ }^{4}$, Robin P Peeters ${ }^{2,3}$.

${ }^{1}$ The Generation R Study Group and Departments of ${ }^{2}$ Internal Medicine, ${ }^{3}$ Rotterdam Thyroid Center, ${ }^{6}$ Epidemiology; Erasmus Medical Center, Rotterdam, The Netherlands Departments of ${ }^{5}$ Clinical Chemistry, ${ }^{4}$ Obstetrics and Gynecology, ${ }^{7}$ Pediatrics; Erasmus Medical Center - Sophia Children's Hospital, Rotterdam, The Netherlands

\section{Introduction}

Suboptimal placental function is associated with pre-eclampsia, fetal growth restriction and premature delivery.

- Thyroid hormone (TH) has a role in the placental development through effects on trophoblast proliferation and invasion.

Thyroid dysfunction has been associated with the pregnancy disorders that arise from compromised placentation.

- We aimed to investigate the clinical association of gestational thyroid function with the placental function, as well as the potential mediation role of placental hemodynamics in the associations of thyroid hormone with gestational outcomes (mediation scheme depicted in Figure 1)

\section{Results}

FT4 was positively linearly associated with umbilical artery PI and uterine artery RI in the second trimester (Figure 1). TSH was negatively linearly associated with uterine artery $\mathrm{RI}$ in the second trimester (Figure 2).

Figure 3 shows the positive association of FT4 with umbilical artery PI in the third trimester. TSH was not associated with umbilical artery PI nor with uterine artery $\mathrm{RI}$ in the third trimester.

Figure 4 shows that with high FT4 there was a higher risk of notching in the uterine artery during the third trimester.

The association of thyroid function with pre-eclampsia and birth weight was partially mediated by placental function; percentage of mediated effect was $13.8 \%$ and $10.4 \%$, respectively (Figure 1 ).

Figure 3. The association of maternal thyroid with the $3^{\text {rd }}$ trimester placental function
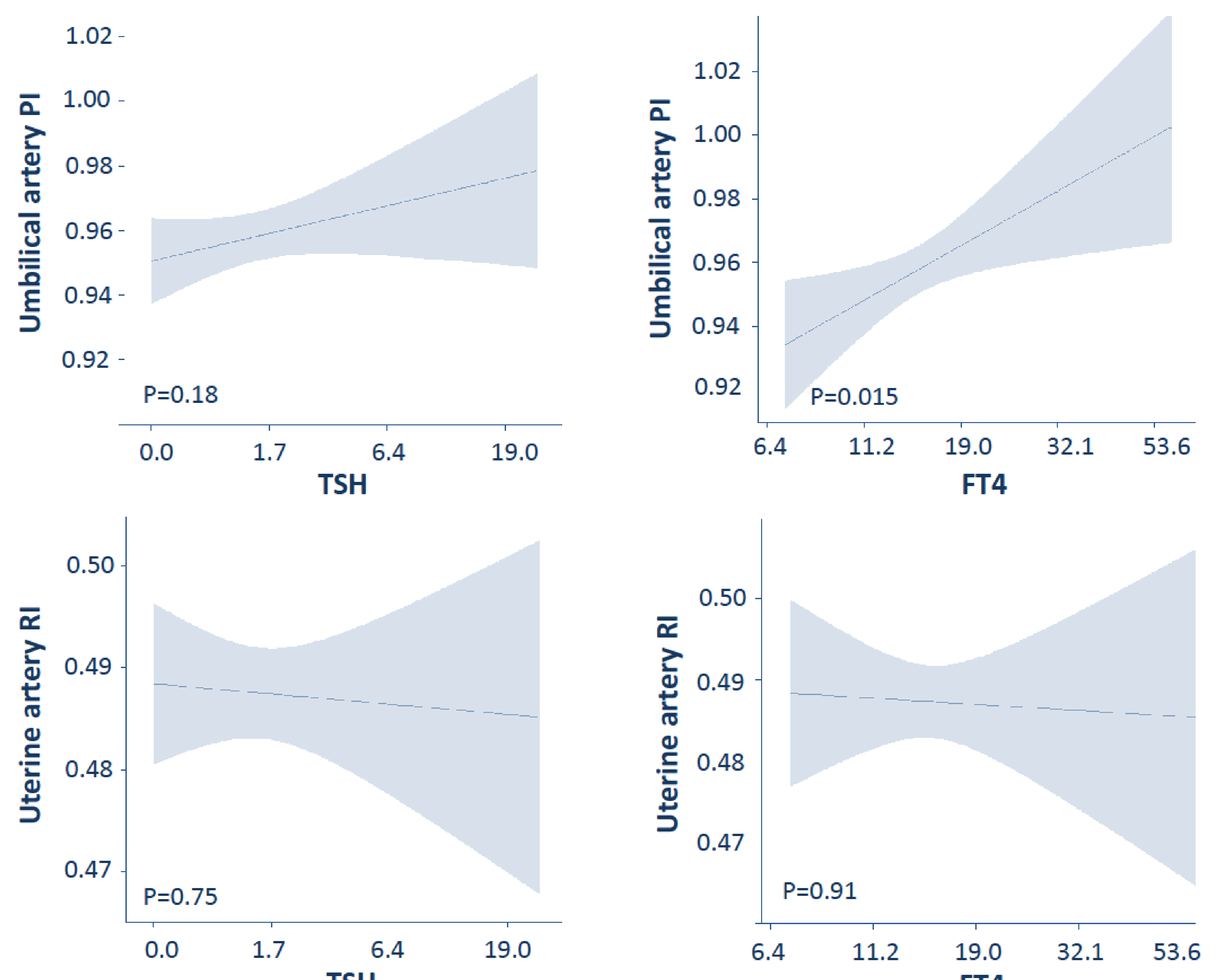

\section{Participants and Methods}

Study was embedded in The Generation R cohort. The population included in the analysis consisted of 5184 pregnant women. Maternal TSH and FT4 were measured in early pregnancy (median 13.4 weeks, 95\% range 9.7-17.6 weeks). Placental function was assessed through vascular resistance measurement by Doppler ultrasound in the umbilical and uterine arteries during second and third trimester. A raised umbilical artery pulsatility index (PI) and uterine artery resistance index (RI) indicate impaired placental function.

Figure 1. The mediation role of the placental function in the association of thyroid function with gestational outcomes

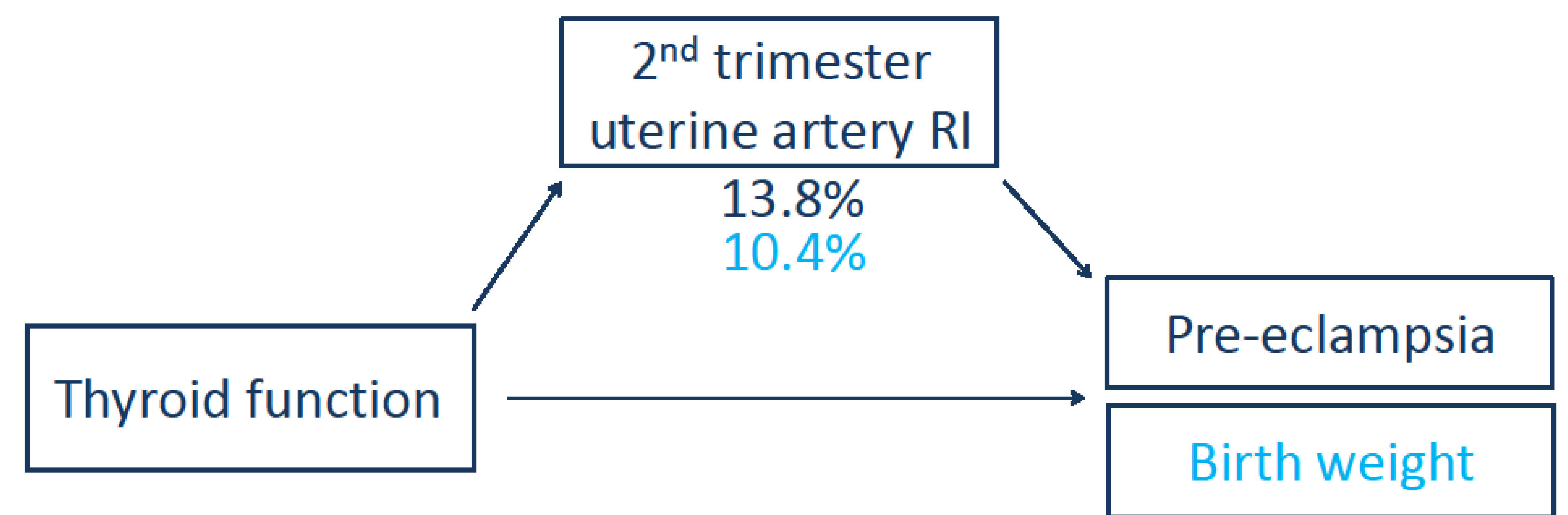

Figure 2. The association of maternal thyroid with the $2^{\text {nd }}$ trimester placental function
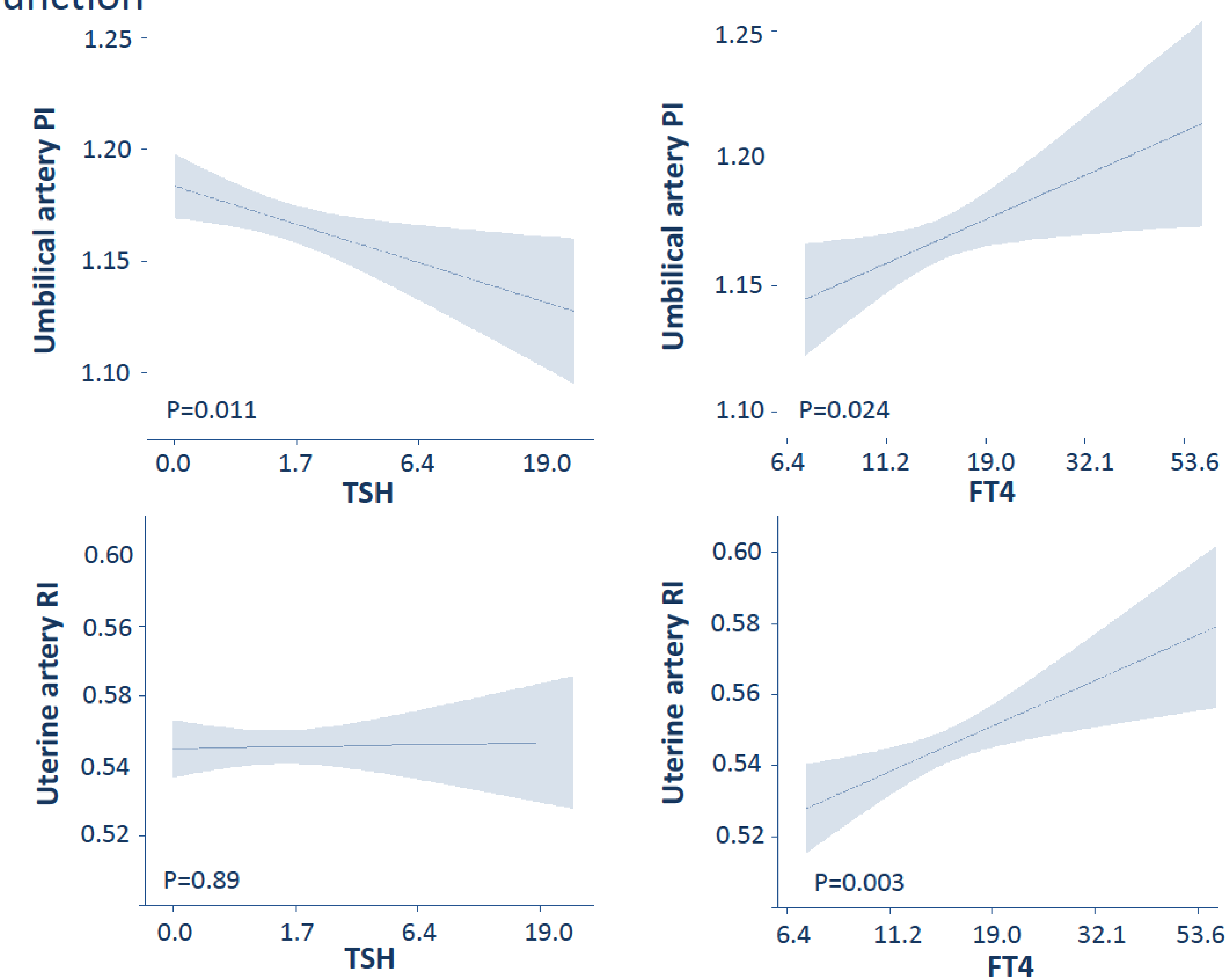

Figure 4. The association of maternal thyroid with the risk of uterine artery notching in the $3^{\text {rd }}$ trimester
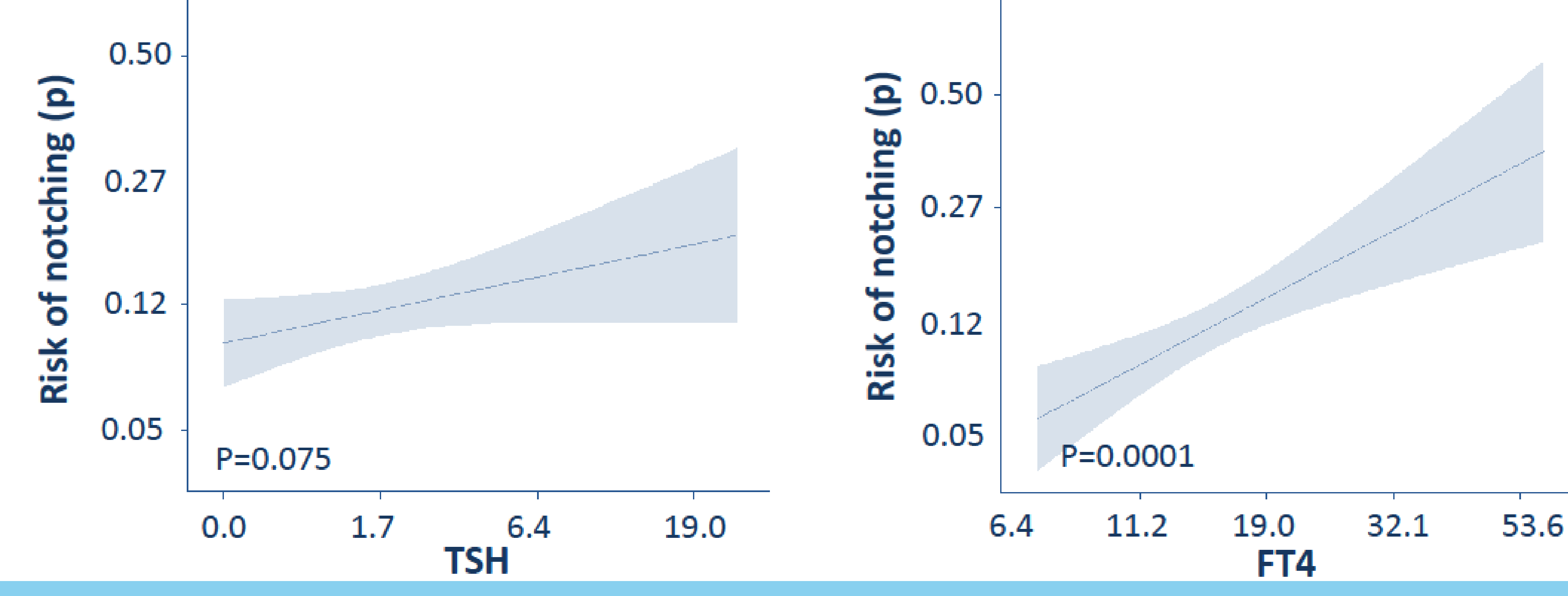

Early gestational thyroid function is associated with placental vascular function later during pregnancy.

High maternal FT4 levels were associated with high placental vascular resistance throughout gestation.

These results suggest that high thyroid function during early pregnancy may increase risk of impaired placentation.

We estimated that $13.8 \%$ and $10.4 \%$ of the association of high thyroid function with pre-eclampsia and birth weight occurs through changes in placental hemodynamics, respectively. 